Research article

\title{
Long-term experience with implanted intrathecal drug administration systems for failed back syndrome and chronic mechanical low back pain JH Raphael*1, JL Southall ${ }^{1}$, TV Gnanadurai1, GJ Treharne ${ }^{3}$ and GD Kitas ${ }^{2}$
}

Address: ${ }^{1}$ Department of Pain Management, Dudley Group of Hospitals NHS Trust, West Midlands, UK, 2 Department of Rheumatology, Dudley Group of Hospitals NHS Trust, West Midlands, UK and ${ }^{3}$ School of Psychology, University of Birmingham, Edgbaston, Birmingham, UK

E-mail: JH Raphael* - JonRaph@AOL.com; JL Southall - julie.hackett@dudleygoh-tr.wmids.nhs.uk; TV Gnanadurai - victorgnanadurai@hotmail.com; GJ Treharne - GJ884@psg-fs4.bham.ac.uk; GD Kitas - g.d.kitas@bham.ac.uk ${ }^{*}$ Corresponding author

Published: 20 June 2002

BMC Musculoskeletal Disorders 2002, 3:17

This article is available from: http://www.biomedcentral.com/I47/-2474/3/17 this notice is preserved along with the article's original URL.

Received: 17 December 200|

Accepted: 20 June 2002

(C) 2002 Raphael et al; licensee BioMed Central Ltd. Verbatim copying and redistribution of this article are permitted in any medium for any purpose, provided

\begin{abstract}
Background: Continuous intrathecal drug delivery has been shown in open studies to improve pain and quality of life in those with intractable back pain who have had spinal surgery. There is limited data on long term effects and and even less for patients with mechanical back pain without prior spinal surgery.
\end{abstract}

Methods: We have investigated spinal drug administration systems for patients with failed back syndrome and chronic mechanical low back pain by patient questionnaire study of the efficacy of this therapy and a case notes review.

Results: 36 patients ( $97 \%$ of 37 approached) completed questionnaires, 24 with failed back syndrome and 12 with chronic mechanical low back pain. Recalled pre-treatment levels with current post-treatment levels of pain and a range of quality of life measures (recorded on I I-point numerical rating scales) were compared. Pain improved significantly in both groups (Wilcoxan signed ranks test, $\mathrm{P}<0.005)$. The majority of quality of life measures improved significantly in the failed back syndrome group (Wilcoxan signed ranks test, $p<0.005$ ) although work interruption and the effect of pain on sex life did not change. There was a trend towards improvement in the majority of quality of life measures in the mechanical back pain group but this did not reach statistical significance due to the smaller numbers in this cohort $(p>0.005$, Wilcoxan signed ranks test with Bonferroni correction).

Diamorphine was used in all 37 patients, bupivacaine in 32, clonidine in 27 and baclofen in 3 . The mean dose of diamorphine increased for the first 2 years but did not change 2-6 years post implant, averaging $4.5 \mathrm{mg} /$ day. Revision surgery was required in $24 \%$ of cases, but reduced to $12 \%$ in the later years of our experience.

Conclusions: We conclude that spinal drug administration systems appear to be of benefit in alleviating pain in the failed back syndrome and chronic mechanical low back pain but need to be examined prospectively. 


\section{Background}

Low back pain is a common complaint with important socio-economic consequences. A comprehensive 1-year survey of back pain in the UK found that there were 14 million general practitioner (GP) consultations, 1.6 million attendances at outpatient clinics, 1 million attendances at physiotherapy, 100,000 hospital admissions, 30,000 day cases and 24,000 operations. It was estimated that the delivery of these services cost 480 million pounds in addition to significant societal costs of lost production ( $€ 3.8$ billion) and social benefits ( $€ 1.4$ billion) [1].

The vast majority of chronic low back pain has no clear cause and is managed symptomatically. Treatment guidelines suggest a multidisciplinary approach starting from the least invasive treatments [2]. Treatments include a range of physical, psychological, medical and surgical therapies, most of which are not clearly substantiated but appear to help some patients. These still leave a significant number of patients with back pain and disability.

The discovery of opioid receptors in the spinal cord led to the rationale for the use of intrathecal morphine for pain relief in cancer [3]. With the development of implantable, programmable, continuous drug delivery systems in the 1980 s, the use of intraspinal opioids was extended to noncancer pain, the commonest of which was back pain; nevertheless, use in such circumstances remains less accepted. This is related to the different outcomes that are strived for in non-cancer pain compared with cancer pain with limited life expectancy. In the case of non-malignant pathology with normal life expectancy, the multidimensional nature of pain and its effects on function and psychological variables such as quality of life and mood are deemed more important. As a result not only pain relief, but also improvements in activities of daily living and psychological well-being are considered important. There are also concerns about drug tolerance and addiction to opioids in cases of normal life expectancy. The initial costs of these therapies are substantial due to capital outlay for the equipment; however, in the longer term there are cost savings due to less healthcare use when the duration of the implantable pump therapy exceeds $12-22$ months [4].

Previous studies of implantable drug delivery systems for patients with back pain have shown benefit [5-7]. However, these studies were restricted to patients who have had prior spinal surgery and have the failed back syndrome (FBS); follow-up was variable and intrathecal drug therapy was mostly limited to opioids alone.

There is a need to define the place of this therapy in those patients with mechanical low back pain without prior surgery (LBP), in addition to those with FBS, to evaluate the place of intrathecal adjuvant analgesics and to measure these effects over a prolonged time period when considering a non-terminal condition.

We have been implanting intrathecal pumps delivering opioid and adjuvant analgesics for chronic back pain including patients without prior spinal surgery at our institution since 1991. We present our experience in terms of the long term effects of this therapy upon (a) pain relief, functioning, quality of life and psychological well-being, as well as (b) drug tolerance and adverse effects.

\section{Methods}

We contacted those patients who had a spinal drug administration system implanted at our pain centre. Patients are selected for this therapy after multidimensional pain assessment and failure to respond to simpler measures including physical, psychological, pharmacological and epidural and facet joint injection therapies. We included only those patients who continued to have the pump refilled by us. This excluded less than $10 \%$ of all patients who had been referred from distant hospitals and had their refills elsewhere. We excluded patients whose pumps were implanted in the last 6 months and sampled only those with a diagnosis of non-malignant back pain.

The sample of patients sent a questionnaire numbered 37, of whom 36 replied (97\% response). The mean age of the responding patients was $54.80 \pm 10.00$ years; 17 (47\%) were male and 19 (53\%) female. The duration of pain complaint prior to the questionnaire was 13.50 years (range 7-35). The spinal implant had been in place for a mean of 4.38 years (range $0.50-9.00$ ). The majority of the pumps used were SynchroMed (Medtronic). At the start of our implant program, Cordis Secor patient-activated pumps were used. In this present sample of 36 patients, 10 had had Cordis Secor pumps implanted, of whom 8 had been subsequently replaced by SynchroMed pumps.

Of the 36 patients, 12 had mechanical low back pain without prior surgery (LBP) and 24 had the failed back syndrome (FBS). The median number of prior spinal operations in the FBS group was 2 (range 1-11) (Table 1).

Patients were sent a questionnaire requesting ratings of pain, the primary endpoint and a range of quality of life measures on 11-point Likert-style numeral rating scales with 0 being defined as absence of the symptom or no interference with the quality of life measure, and 10 being defined as maximum imaginable level of the symptom or maximal imaginable interference with the quality of life measure $[8,9]$. Patients were asked to give ratings on all variables for their current situation (post-treatment) and their condition as recalled before they had received the spinal implant (pre-treatment). 


\begin{tabular}{llll}
\hline & Total Sample & LBP & FBS \\
\cline { 3 - 4 } & & $\mathrm{n}=36$ & $\mathrm{n}=12$ \\
\hline & & & \\
Sex (male:female) & & $5: 7$ & $12: 12$ \\
Age mean (SD) (in years) & $17: 19$ & $56.33(12.32)$ & $54.00(8.75)$ \\
Pain duration mean (SD) (in years) & $54.80(10.00)$ & $8.89(2.57)$ & $15.55(7.04)$ \\
Implant duration mean (SD) (in years) & $13.61(6.77)$ & $2.18(2.05)$ & $5.48(2.85)$ \\
Previous spinal operations median (range) & $4.38(3.03)$ & & $2(1-11)$
\end{tabular}

LBP = Low Back Pain (without prior surgery); FBS = Failed Back Syndrome

Pain was investigated both by asking level of pain experienced, the level of pain relief and also the duration of relief in the average day. Pain was also rated on a 4-point verbal scale of its severity (none, mild, moderate or severe).

Psychological attributes of the pain experience were measured by investigating levels of depression, overall quality of life, coping ability and dependency on others. Sociological dimensions were measured by inquiring about interruption to work, social life, sex life, mobility, driving and housework in a similar fashion.

The questionnaire also requested comments on use of analgesics, average number of general practitioner (GP) visits and inquired about satisfaction with the treatment.

The identities of the patient questionnaires were only known to a third party until the data was analysed. The identities of the data were then determined and information on the patients' diagnosis, spinal implantation and subsequent care were collected from case records.

Differences between the LBP and FBS groups were calculated using Mann Whitney U tests and independent sample t-tests. Compared to the LBP group, the FBS group reported longer duration since they started experiencing pain (means 8.89 (2.57 and 15.55 (7.04, respectively; $\mathrm{t}(29)=2.74, \mathrm{P}<0.01$ ) and since implantation (means 2.18 (2.05 and 5.48 (2.85, respectively; $\mathrm{t}(34)=3.57, \mathrm{P}<$ $0.001)$, but did not differ in age (Table 1). No significant differences existed between these groups on any of the pain or quality of life questionnaire measures, but as the groups were at different stages of coping with back pain they were analysed as separate groups.

Relationships between aspects of the benefits of treatment were analysed by Spearman's nonparametric correlations. Differences between pre- and post-treatment scores were analysed using chi-squared tests, Wilcoxon signed ranks tests, and paired samples t-tests. A Bonferroni adjustment to the level of significance was made to account for the number of tests carried out between pre- and post-treatment scores with significance at $\mathrm{p}<0.005$.

\section{Results}

\section{Response to treatment}

Overall, the treatment was considered to be worthwhile by the patients, with 26 (74\% of the valid 35) responding that it was very worthwhile, $5(14 \%)$ responding that it was quite worthwhile, $3(9 \%)$ responding that it was adequate and only $1(3 \%)$ responding that it was not worthwhile. The LBP group were on the lower side of these scores with 7 (64\% of the valid 11$)$ responding that it was very worthwhile, 2 (19\%) responding that it was quite worthwhile, $1(9 \%)$ responding that it was adequate and this group included the $1(9 \%)$ who responded that it was not worthwhile.

Analyses of the Spearman's correlations of all pain and psychosocial questionnaire items across both the FBS and LBP groups indicate that on average they were only moderately related (within the same time of reference), with a mean of 0.38 (equivalent to $\mathrm{P}<0.05$ ), from a range of 0.00 (NS) - $0.86(\mathrm{P}<0.001)$. However, as these variables were considered to be conceptually independent, further analyses were carried out on the individual items, rather than bluntly grouping all scores.

Across all analyses of pre- and post-treatment scores, beneficial change was seen in the majority of cases on most variables: the median number of detrimental changes per variable was 2 , with a range of $1-5$. Tied scores were only moderately common, with a median number of 5 per variable, from a range of $0-19$. For the FBS group, the median number of detrimental changes per variable was 1 , with a range of $0-4$. The median number of ties was 4 per variable, from a range of $0-14$. For the LBP group, the median 
number of detrimental changes per variable was 0 , with a range of $0-2$. The median number of ties was 1 per variable, from a range of $0-5$.
Differences between pre- and post-treatment scores are shown for the FBS group in Table 2 and for the LBP group in Table 3.

Table 2: Efficacy of intrathecal implants in failed back syndrome ( $n$ between 20 and 24)

\begin{tabular}{|c|c|c|c|c|c|}
\hline Dimension & Variable & $\begin{array}{l}\text { Pre-Treatment } \\
\text { Median (Quartiles) }\end{array}$ & $\begin{array}{l}\text { Post-Treatment } \\
\text { Median (Quartiles) }\end{array}$ & Z Scorea & $P$ valueb \\
\hline \multirow[t]{2}{*}{ Sensory } & Pain & $9.50(8.00,10.00)$ & $5.00(4.00,6.00)$ & 4.22 & $<0.00006^{*}$ \\
\hline & Pain Relief & $9.00(8.00,10.00)$ & $3.50(2.75,6.25)$ & 4.01 & $<0.00006^{*}$ \\
\hline \multirow[t]{4}{*}{ Psychological } & Depression & $9.00(7.00,10.00)$ & $4.00(0.00,6.00)$ & 4.01 & $<0.00006^{*}$ \\
\hline & Quality of Life & $10.00(8.00,10.00)$ & $5.00(3.00,7.75)$ & 3.79 & $<0.00022^{*}$ \\
\hline & Coping & $8.00(7.00,10.00)$ & $5.00(4.00,7.00)$ & 3.68 & $<0.00032^{*}$ \\
\hline & Dependency & $8.00(6.00,10.00)$ & $6.00(3.00,8.00)$ & 3.09 & $<0.0027^{*}$ \\
\hline \multirow[t]{7}{*}{ Sociological } & Sleep & $9.50(8.00,10.00)$ & $6.00(4.00,9.00)$ & 3.06 & $<0.0027^{*}$ \\
\hline & Work & $10.00(9.75,10.00)$ & $10.00(8.25,10.00)$ & 1.20 & 0.2301 \\
\hline & Social Life & $10.00(9.00,10.00)$ & $6.50(4.00,8.00)$ & 3.25 & $<0.00137^{*}$ \\
\hline & Sex Life & $9.50(8.25,10.00)$ & $8.50(5.00,10.00)$ & 2.12 & 0.0340 \\
\hline & Driving & $5.00(2.00,9.00)$ & $2.00(0.00,6.00)$ & 3.32 & $<0.00097^{*}$ \\
\hline & Housework & $10.00(8.00,10.00)$ & $6.00(4.00,8.00)$ & 3.83 & $<0.00014^{*}$ \\
\hline & Mobility & $10.00(9.00,10.00)$ & $6.50(5.25,9.00)$ & 3.01 & $<0.0027^{*}$ \\
\hline
\end{tabular}

a The $\mathrm{Z}$ score is converted from the Wilcoxon signed ranks test. ${ }^{b} \mathrm{~A}$ Bonferroni adjustment for the number of tests indicates $\mathrm{P}<0.005(0.05 / 10)$ as significant.

Table 3: Efficacy of intrathecal implants in low back pain with out prior surgery ( $\mathrm{n}$ between 9 and II)

\begin{tabular}{|c|c|c|c|c|c|}
\hline Dimension & Variable & $\begin{array}{l}\text { Pre-Treatment } \\
\text { Median (Quartiles) }\end{array}$ & $\begin{array}{l}\text { Post-Treatment } \\
\text { Median (Quartiles) }\end{array}$ & Z Score ${ }^{a}$ & $P$ valueb \\
\hline \multirow[t]{2}{*}{ Sensory } & Pain & $9.00(8.00,10.00)$ & $6.00(4.00,6.00)$ & 2.87 & $0.0041^{*}$ \\
\hline & Pain Relief & $8.00(6.50,9.50)$ & $4.00(1.50,6.00)$ & 2.54 & 0.0111 \\
\hline \multirow[t]{4}{*}{ Psychological } & Depression & $8.00(5.50,10.00)$ & $5.00(2.00,6.00)$ & 2.37 & 0.0178 \\
\hline & Quality of Life & $9.50(8.00,10.00)$ & $6.50(3.75,7.25)$ & 2.68 & 0.0074 \\
\hline & Coping & $8.50(7.75,10.00)$ & $6.00(2.50,7.00)$ & 2.82 & $0.0048^{*}$ \\
\hline & Dependency & $8.00(7.00,9.00)$ & $6.00(5.75,6.50)$ & 2.72 & 0.0065 \\
\hline \multirow[t]{7}{*}{ Sociological } & Sleep & $10.00(9.00,10.00)$ & $7.50(4.50,9.00)$ & 2.68 & 0.0074 \\
\hline & Work & $10.00(8.50,10.00)$ & $9.00(6.50,10.00)$ & 1.84 & 0.0658 \\
\hline & Social Life & $10.00(9.00,10.00)$ & $8.00(6.00,8.00)$ & 2.71 & 0.0067 \\
\hline & Sex Life & $9.50(7.25,10.00)$ & $9.00(4.75,10.00)$ & 1.09 & 0.2757 \\
\hline & Driving & $7.00(3.00,8.00)$ & $7.00(0.00,8.00)$ & 0.37 & 0.7114 \\
\hline & Housework & $9.00(7.00,10.00)$ & $6.00(5.00,8.00)$ & 1.50 & 0.1336 \\
\hline & Mobility & $9.00(7.00,10.00)$ & $8.00(6.00,8.00)$ & 2.84 & $0.0045^{*}$ \\
\hline
\end{tabular}

a The $\mathrm{Z}$ score is converted from the Wilcoxon signed ranks test. ${ }^{b} \mathrm{~A}$ Bonferroni adjustment for the number of tests indicates $\mathrm{P}<0.005(0.05 / \mathrm{I} 0)$ as significant. 
The FBS group showed significant improved on the majority of measures: pain score on the 11-point system reduced from a median of 9.50 to 5 (Wilcoxon $\mathrm{W}=276.00$, $\mathrm{Z}=4.22, \mathrm{P}<0.00006)$. Furthermore, verbal assessment of pain most commonly changed from severe to moderate, and when these ratings were converted to a 4-point numerical system, the improvement was significant (Wilcoxon $\mathrm{W}=136.00, \mathrm{Z}=3.64, \mathrm{P}<0.0003)$. Pain relief interruption on the 11-point system decreased from a median of 9 to 3.50 (Wilcoxon $\mathrm{W}=251.50, \mathrm{Z}=4.07, \mathrm{P}<$ $0.00006)$.

With respect to the psychological dimension of pain experience, for the FBS group the effect of pain on depression reduced from a median of 9 pre-treatment to 4 post-treatment (Wilcoxon $\mathrm{W}=249, \mathrm{Z}=4.01, \mathrm{P}<0.00022$ ); on quality of life from a median of 10 to 5 (Wilcoxon $W=189.00$, $\mathrm{Z}=3.79, \mathrm{P}<0.00022)$; on coping from a median of 8 to 5 (Wilcoxon $\mathrm{W}=258.50, \mathrm{Z}=3.68, \mathrm{P}<0.00032$ ); and on dependency from a median of 8 to 6 (Wilcoxon $\mathrm{W}=$ 171.50, $\mathrm{Z}=3.09, \mathrm{P}<0.0027)$.

In the sociological dimension of pain experience, for the FBS group the effect of pain on sleep reduced from a median of 9.50 pre-treatment to 6 post-treatment (Wilcoxon $\mathrm{W}=186.50, \mathrm{Z}=3.06, \mathrm{P}<0.0027)$; on work, there appeared to be no effect, with both medians being 10 (Wilcoxon $\mathrm{W}=26.50, Z=1.20$, exact $\mathrm{P}=0.2301)$; on social life, the effect reduced from a median of 10 to 6.50 (Wilcoxon $\mathrm{W}=175.50, \mathrm{Z}=3.25, \mathrm{P}<0.00137)$; on sex life, there appeared to be no effect, with the median changing from 9.50 pre-treatment to 8.50 post-treatment (Wilcoxon $\mathrm{W}=86.00, \mathrm{Z}=2.12$, exact $\mathrm{P}=0.034)$; on driving, the effect reduced from a median of 5 to 2 (Wilcoxon $\mathrm{W}=$ 105.00, $\mathrm{Z}=3.32, \mathrm{P}<0.00097)$; on housework from a median of 10 to 6 (Wilcoxon $\mathrm{W}=207.00, \mathrm{Z}=3.83, \mathrm{P}<$ $0.00014)$; and on mobility from a median of 10 to 6.50 (Wilcoxon $\mathrm{W}=201.00, \mathrm{Z}=3.01, \mathrm{P}=0.0027$ ).

For the LBP group, there was a significant reduction in pain score with treatment from a median of 9 to 6 (Wilcoxan, $\mathrm{Z}=2.87, \mathrm{p}=0.0041$ ); however, although quality of life measurement changes were similar to the failed back syndrome cohort and showed a trend to improvement this was not statistically significant (Wilcoxan, p > 0.005).

Although the results for work disruption do not look as good as for the other aspects (due to the high number of ties ( $n=19$ of the 31 valid cases) on this variable), of the 15 patients less than 50 years of age, none were able to work before receiving the implant, but 4 (27\%) were able to return to work after implantation.

For the FBS group, the number GP visits per month reduced significantly from 2.24 pre-treatment to 0.72 post- treatment (Wilcoxon $\mathrm{W}=136.00, \mathrm{Z}=3.53$, adjusted $\mathrm{P}<$ $0.05)$, whereas for the LBP group, the median number reduced from 2.07 pre-treatment to 0.90 post-treatment, but again this did not reach significance (Wilcoxon $\mathrm{W}=$ 6.00, $Z=1.60$, exact $P>0.1$ ).

\section{Pharmacological data}

All 37 patients received diamorphine, 32 received bupivacaine, 27 clonidine and 3 baclofen.

The doses of diamorphine over time are shown in Table 4. There was wide spread and the median dose increased over the first two to three years after implant. The data we have beyond this is restricted to the FBS group, for whom the dose plateaued from two to six years at a median of 4.5 $\mathrm{mg} /$ day. Rapidly increasing dose to reach a daily dose of $10 \mathrm{mg} /$ day occurred in 4 patients. Higher diamorphine doses were used in the FBS group at similar time points (Table 5).

Table 4: Diamorphine dose median (range) (in mg)

\begin{tabular}{lll}
\hline & LBP & FBS \\
\cline { 2 - 3 } Years After Implant & $\mathrm{n}=12$ & $\mathrm{n}=25$ \\
& & \\
\hline & & \\
2 & $2.30(0.75-4.00)$ & $3.10(0.50-3.75)$ \\
3 & $3.30(0.75-6.00)$ & $5.20(0.75-10.00)$ \\
4 & $4.30(0.75-7.50)$ & $5.30(0.75-10.00)$ \\
5 & & $5.20(0.75-16.00)$ \\
6 & & $5.50(0.75-16.00)$ \\
& & $4.90(0.75-16.00)$ \\
\hline
\end{tabular}

LBP = Low Back Pain (without prior surgery); FBS = Failed Back Syndrome

Table 5: Comparison of drugs in spinal implant system in LBP and FBS

$\begin{array}{ll}\text { LBP } & \text { FBS } \\ n=12 & n=25\end{array}$

$\begin{array}{lll}\text { Diamorphine n (\%) } & 12(100) & 25(100) \\ \text { Bupivacaine n (\%) } & 9(75) & 23(92) \\ \text { Clonidine n (\%) } & 8(67) & 19(76) \\ \text { Baclofen n (\%) } & 0(0) & 3(12)\end{array}$

LBP = Low Back Pain (without prior surgery); FBS = Failed Back Syndrome 


\section{Complications}

There were 10 episodes of revision surgery, due to catheter problems (3), pump position problems (4), infection (2) and cerebrospinal fluid leak (1) (Table 6).

Table 6: Complications of intrathecal pump implant

\begin{tabular}{lll}
\hline Period & $\begin{array}{l}\text { Type of } \\
\text { Complication }\end{array}$ & $\begin{array}{l}\text { Number of } \\
\text { Occurrences }\end{array}$ \\
\hline 1991-1995 $(n=20)$ & Revision surgeries & 8 \\
& Infections & 5 \\
$1996-2000(n=17)$ & Revision surgeries & 2 \\
& Infections & 0 \\
Total Revision Surgeries $(n=10)$ & Catheter-related & 3 \\
& $\begin{array}{l}\text { Pump-related } \\
\text { Infections requir- }\end{array}$ & 4 \\
& ing surgery & \\
& Cerebrospinal fluid & 1 \\
& leak & \\
\hline
\end{tabular}

There were a total of 5 documented infections after implant ( 2 of which required surgery, whilst the others managed with antibiotics).

Comparing complications during the first half ( 4.5 years) of our experience with the second 4.5 years, revisions fell from 5 out of 22 to 2 out of 17 and there have been no infections in the second half (chi squared $=7.5, \mathrm{p}<0.01$ ).

\section{Discussion}

The pain generators in chronic low back pain are various and often not clearly definable, but comprise a combination of nociceptive and neuropathic components. Management of chronic back pain is thus to an extent empirical and commonly involves a stepwise progression from the lowest risk treatments [2]. As a result, patients who have failed to find relief may eventually come to be treated on a trial basis with oral opiates; however, a study of long term oral opioids for low back and neuropathic pain, found good results in only $16.7 \%$ of cases [10]. This limitation is most obvious in neuropathic pain states, which are less opioid sensitive [11]. The pathological changes consequent upon nerve injury such as up-regulation of cholecystokinin receptors shifts the opioid doseresponse curve to the right. Spinal opioid delivery allows for a higher concentration at the receptors on primary afferents at the dorsal horn than can be achieved by systemic administration since the latter is often limited by dose dependent side effects. In an animal study of neuropathic pain using the sciatic nerve constriction model, Suzuki et al. [12] showed that greater analgesia could be achieved with opioids delivered intrathecally rather than systemically.

Spinal opioids for low back pain is a therapy of last resort and is controversial. Tutak et al. [13] reported on concerns about opiate tolerance with continually increasing doses over an 18-month period and Paice et al. [5] found a mean opioid dose of $9.6 \mathrm{mg} /$ day of morphine after only one year. In contrast, Winkelmuller and Winkelmuller [6] found a mean dose of $5.6 \mathrm{mg} /$ day of morphine at an average follow up of 3.4 years and drug tolerance was uncommon. Nevertheless, concerns about opiate tolerance remain. There is retrospective outcome data that describes improvements in pain and a range of quality of life measures with this therapy $[5,6,13]$; however, this is largely of modest duration, has not included patients with low back pain who have not had prior spinal surgery and the vast majority of patients received spinal opioids alone.

We have a long experience at our institution of implanting intrathecal drug administration systems for back pain, with or without prior spinal surgery and of delivering combinations of drugs that include opiates and adjuvant analgesics. We evaluated the efficacy of this therapy upon pain and quality of life and investigated opiate tolerance and adverse effects. Patients selected for intrathecal drug implants had failed to gain pain relief with simpler measures including drugs, physical treatments, psychological therapies and spinal injections according to the recognised criteria for spinal implantation [2]. We have found the pain level reduced by 3 or more on an 11-point scale, a clinically significant amount, with an average implant duration of 4.38 years. Most of the quality of life measures recorded showed a clinically significant improvement with therapy which reached statistical significance in the cohort with failed back syndrome. The smaller sample size preventing the majority quality of life effects reaching statistical significance for the mechanical low back pain group. Measures of the psychological dimension of pain experience (depression, quality of life, coping, dependency on others) all improved in both groups. All reached statistical significance for the failed back syndrome cohort but only in the case of coping for the mechanical back pain group. Measures of the sociological dimension of pain experience (sleep, social life, driving, housework) also showed significant improvement, with the exception of the effect on sex life and work which were not shown to have been improved. These changes reached statistical significance in the failed back syndrome group. However, of the 15 patients under 50 years old, all of whom had not worked preceding implant, 4 had since returned to employment. Thus an externally reliable measure of work ability improved. 
Diamorphine was used in all our patients and bupivacaine and clonidine used in approximately two thirds. The median diamorphine dose increased over the first few years after implant but in those patients with longer term dosage data, the FBS group, we found that the median dose did not change significantly between 2 and 6 years post implant averaging $4.5 \mathrm{mg} /$ day. Only 4 patients showed a continually rising dose requirement of diamorphine to reach $10 \mathrm{mg} /$ day and none had shown evidence of opiate abuse. Thus we did not find tolerance to diamorphine in this patient group to be a common occurrence. The dose of diamorphine was greater in the FBS group at comparable time points compared to those without prior spinal surgery, and there was a significantly greater use of bupivacaine and clonidine in FBS compared with non-operated low back pain. This may reflect the greater proportion of neuropathically-mediated pain following spinal surgery, presumably from scar tissue.

There was no significant difference in outcome scores between those with FBS and those who had not had prior spinal surgery both showing in pain level and in the majority of quality of life measures; however, the numbers in the mechanical back pain group were insufficient for the majority of quality of life measures to reach statistical significance. There are no previous studies including patients with non-operated low back pain. They comprise an important group, partly because the condition is common and often otherwise intractable, but also because the criteria for spinal surgery in this group have narrowed with time. Indeed in our practice we are seeing more patients with non-operated low back pain and less with FBS compared with earlier practice.

The overall complication rate requiring revision surgery was comparable to others $[5,6,14]$; however, this reduced significantly with experience.

A retrospective design relies upon patient recall. Several studies suggest that patients with chronic pain contrast with those with acute pain in having a more reliable recall of past pain [15,16] and its affective dimensions [17]. Patients can over-rate past pain [18] and thus overestimate benefits of a treatment; however, overestimates seem to occur particularly when pain measures have increased over the treatment period which was not the case in this study [19]. Pain was our primary endpoint, and as a subjective experience is difficult to measure. Rating scales are commonly used for their simplicity and associated compliance. We chose to use numerical rating scales as these compare favourably against visual analogue and other rating scales in terms of minimising scoring errors and optimising sensitivity [8].
We included in our questionnaire a range of questions to cover the sensory, affective and cognitive dimensions of pain experience. Many of the questionnaires of these dimensions are limited by a complexity that diminishes patient compliance. Our high response rate may have been achieved with the simpler numerical rating scales we employed. Similar scales have been found to have high internal consistency although their validity has not been properly evaluated [9].

We collected data on the use of health care resources since overuse of such resources is a reason for referral. In this area there are no established measures. We used GP visits, which can involve any interaction even if the original pain problem is addressed. As such, an unchanged rate of visiting cannot be easily interpreted, but the reduction in GP visits that we found may be meaningful.

Our results are comparable to those of others. Winkelmuller and Winkelmuller [6] found greater pain reduction of just over $50 \%$ on a visual analogue scale; however, their sample was highly selected as a fifth of patients originally implanted were later explanted due most commonly to poor pain relief and not included in their assessment. Paice et al. [5] described a $60 \%$ degree of pain relief; however, this was based upon physician reports of patients pain relief. Of the fraction of patients they contacted, it appeared that physicians had overestimated the degree of pain relief. Others have found improvements in activities of daily living $[5,6]$; however, the methods used to assess these do not allow for closer comparisons.

Other published series used adjuvant analgesics far less frequently than we have. In Paice et al.'s survey [5], only $19 \%$ of cases included a local anaesthetic and none clonidine or baclofen. In Winkelmuller and Winkelmuller's study [6], clonidine was used in only 2 of the 82 cases. We have not noted any serious complications with a greater use of these adjuvant analgesics, and the opioid doses we have used are considerably lower than in these other studies, thus the greater use of adjuvant analgesics appears to have an opioid-sparing effect. Furthermore, opioid tolerance has not been a common clinical problem in our experience.

Pains with a significant neuropathic component appear opioid responsive in this study which concurs with others [5]. Indeed, intrathecal use of opioids in this group may have distinct advantages as it obviates many of the side effects of systemic opioids that may be required in large doses producing unacceptable side effects.

Spinal analgesia has limitations. In vivo experiments suggest differential sensitivity between supraspinal and spinal sites with the supra being more sensitive possibly 
because multiple synaptic connections are more sensitive to opioid than oligosynaptic ones [20]. Although with direct spinal application of opioids patients can achieve analgesia they may not achieve the dissociation from their pain with the systemic doses of morphine that were too low to have a detectable effect at the spinal level. The multidimensional model of pain draws attention to role of higher centres as well as the dorsal horn in pain processing and in clinical practice this perhaps explains the variation in response of patients to systemic compared to spinal opioids in their pain management and is the basis of spinal drug testing before committing to long term delivery systems.

To our knowledge, this is the longest survey of implanted intrathecal pumps for pain and the first to address low back pain without prior surgery. Using a retrospective questionnaire we have found an improvement in pain and range of quality of life measures over a prolonged time averaging 4.38 years (range $0.50-9.00$ years). Tolerance to intrathecal opioids does not appear to be a significant problem and the use of bupivacaine and clonidine together with diamorphine is without complications and appears to have an opioid-sparing effect. Revision surgery especially for catheter-related problems continues to be a common problem with the current technology, but complication rates in our study were dependent upon operator and unit experience.

Intrathecal drug delivery systems for failed back syndrome and chronic mechanical low back pain unresponsive to simpler measures appears a reasonable therapeutic option, but there is need to confirm this in prospective randomised controlled trials.

\section{Competing interests}

None declared

\section{Authors' contributions}

Author 1 (JHR) designed the study, participated in analysis of the data, drafted the manuscript

Author 2 (JLS) identified patient sample, collated the questionnaires

Author 3 (TVG) participated in questionnaire design

Author 4 (GJT) participated in analysis of the data

Author 5 (GDK) revised the manuscript

\section{References}

I. Clinical Standards Advisory Group on Back Pain. HMSO 1994

2. Krames ES: Intraspinal opioid therapy for chronic non-malignant pain: current practice and clinical guidelines. J Pain Symptom Manage 1996, I 1:333-52
3. Wang JK, Nauss LA, Thomas JE: Pain relief by intrathecally applied morphine in man. Anesthesiology 1979, 50:|49-5 I

4. Lissovoy G, Brown RE, Halpern M, Hassenbusch SJ, Ross E: Cost-effectiveness of long-term intrathecal morphine therapy for pain associated with failed back surgery syndrome. Clinical Therapeutics 1997, 19(1):96-1 I2

5. Paice JA, Penn RD, Shott S: Intraspinal morphine for chronic pain: a retrospective multicenter study.J Pain Symptom Manage 1996, I I:7|-80

6. Winkelmuller M, Winkelmuller W: Long-term effects of continuous intrathecal opioid treatment in chronic pain of non-malignant etiology. I Neurosurg 1996, 85:458-67

7. Anderson VC, Burchiel KJ: A prospective study of long term intrathecal morphine in the management of chronic non-malignant pain. Neurosurgery 1999, 44:289-300

8. Jensen MP, Karoly P, Braver S: The measurement of clinical pain intensity: a comparison of six methods. Pain 1986, 27: I 17-26

9. Jensen MP, Karoly P, Harris P: Assessing the affective component of chronic pain: development of the Pain Discomfort Scale. J Psychosom Res 1991, 35: 149-54

10. Zenz M, Strumpf M, Tryba M: Long term oral opioid therapy in patients with chronic nonmalignant pain. I Pain Symptom Manage 1992, 7:69-77

II. Jadad AR, Carroll D, Glynn CJ, Moore RA, McQuay HJ: Morphine responsiveness of chronic pain: double-blind randomised crossover study with patient-controlled analgesia. Lancet 1992, 339: | 367-7|

12. Suzuki $\mathrm{R}$, Chapman $\mathrm{V}$, Dickenson $\mathrm{AH}$ : The effectiveness of spinal and systemic morphine on rat dorsal horn neuronal responses in the spinal nerve ligation model of neuropathic pain. Pain 1999, 80:215-28

13. Tutak $U$, Doleys DM: Intrathecal infusion systems for treatment of chronic low back pain of non-cancer origin. South Med J 1 996, 89:295-300

14. Follett KA, Naumann CP: A prospective study of catheter-related complications of intrathecal drug delivery systems. J Pain Symptom Manage 2000, 19:209-15

15. Bolton JE: Accuracy of recall of usual pain intensity in back pain patients. Pain 1999, 83:533-9

16. Linton SJ, Gotestam KG: A clinical comparison of two pain scales: correlation, remembering chronic pain, and a measure of compliance. Pain 1983, 17:57-65

17. Edwards L, Pearce S, Collett BJ, Pugh R: Selective memory for sensory and affective information in chronic pain and depression. Br J Clin Psychol 1992, 3 I:239-48

18. Erskine A, Morley S, Pearce S: Memory for pain: a review. Pain 1990, 41:255-65

19. Bryant RA: Memory for pain and affect in chronic pain patients. Pain 1993, 54:347-51

20. Hill RG, Salt TE, Pepper CM: A comparison of the effectiveness of intravenous morphine at attenuating the nociceptive responses of medullary dorsal horn and thalamic neurones. Life Sci | 982, 3 I:233|-34

\section{Pre-publication history}

The pre-publication history for this paper can be accessed here:

http://www.biomedcentral.com/1471-2474/3/17/prepub 